\title{
Development of High Resolution Gamma-Detectors for PET
}

\author{
A. Kobayashi, E. Yusaku, H. Ito, H. Kawai, T. Mizuno, F. Kento, S. Kimura
}

\section{INTRODUCTION}

$\mathrm{R}$ ECENTLY we are developing a new gamma ray detector using wavelength-sifting-fibers (WLSFs) and scintillator plate $(34 \mathrm{~mm} \times 34 \mathrm{~mm} \times 4 \mathrm{~mm})$ for Positron Emission Tomography (PET) [1]. It is expected the position resolution and the energy resolution are a few $\mathrm{mm}$ [2] and approximately $10 \%$ [3], respectively. Mainly the detector has two advantages. First, it can reduce the expense of cutting scintillator into small pieces. Second, it probably achieves the high position resolution by using $0.2 \mathrm{~mm}$ phi WLSFs.

In this study, the detector performance for $511-\mathrm{keV}$ gamma ray is estimated by an experiment.

\section{CONCEPT OF DETECTOR}

The detector consists of six plate detectors (the effective area is $300 \mathrm{~mm} \times 300 \mathrm{~mm}$ ) setting up around the human body. The plate detector consists of 8 layers. The structure of a layer is shown in Fig.1.

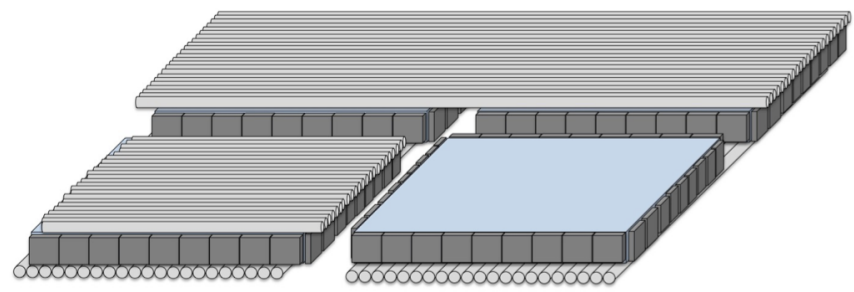

Fig. 1. A schematic of a layer sample which consist of $2 \times 2$ scintillator plates. WLSFs attached to the surface and SiPMs attached to the side.

Each layer consists of 8 times 8 scintillator plates. The size of each scintillator plate is $34 \mathrm{~mm} \times 34 \mathrm{~mm} \times 4 \mathrm{~mm} .160$ wavelength-sifting fibers (WLSFs) cover each $34 \mathrm{~mm} \times 34$ $\mathrm{mm}$ surface of each plate along each axis ( $\mathrm{x}$ or $\mathrm{y}$ ). Ends of

This Fullpaper is submitted June 1, 2017.

Atsushi Kobayashi is with Graduate School of Science and Engineering,

Chiba University, Chiba, Japan (e-mail: koba@hepburn.s.chiba-u.ac.jp).

Yusaku Emoto is with Graduate School of Science, Chiba University,

Chiba, Japan (e-mail: yusaku@hepburn.s.chiba-u.ac.jp).

Hiroshi Ito is with Graduate School of Science, Chiba University, Chiba, Japan (e-mail: hiroshi@hepburn.s.chiba-u.ac.jp).

Hideyuki Kawai is with Graduate School of Science, Chiba University,

Chiba, Japan (e-mail: kawai@hepburn.s.chiba-u.ac.jp).

Takahiro Mizuno is with Graduate School of Science, Chiba University,

Chiba, Japan (e-mail: mizuno@hepburn.s.chiba-u.ac.jp).

Shota Kimura is with Graduate School of Science, Chiba University, Chiba, Japan (e-mail: shota@hepburn.s.chiba-u.ac.jp).

Kento Hujihara is with Graduate School of Science, Chiba University, Chiba, Japan (e-mail: kento@hepburn.s.chiba-u.ac.jp).
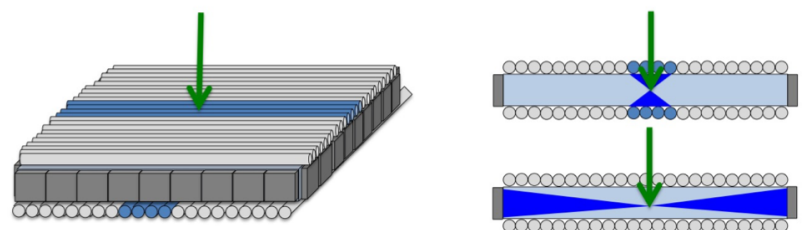

Fig. 2. The method of gamma ray detection.

each fiber are connected to silicon photomultipliers (SiPMs). On each side $(34 \mathrm{~mm} \times 3.4 \mathrm{~mm}$ surface $)$ of plate $10 \mathrm{SiPMs}$ are glued. (Fig.1)

Fig. 2 shows a method of gamma ray detection. When a gamma incidents to the plate and deposit energy, the light emitted. The light satisfied total reflection condition propagates WLSFs, detected by SiPMs connected end of the fiber and the incident position of gamma is measured. On the other hands, the light leaked out from the crystal detected by glued SiPMs on side. Then deposit energy and incident time of gamma are measured.

\section{EXPERIMENT}

The setup is shown in Fig.3.
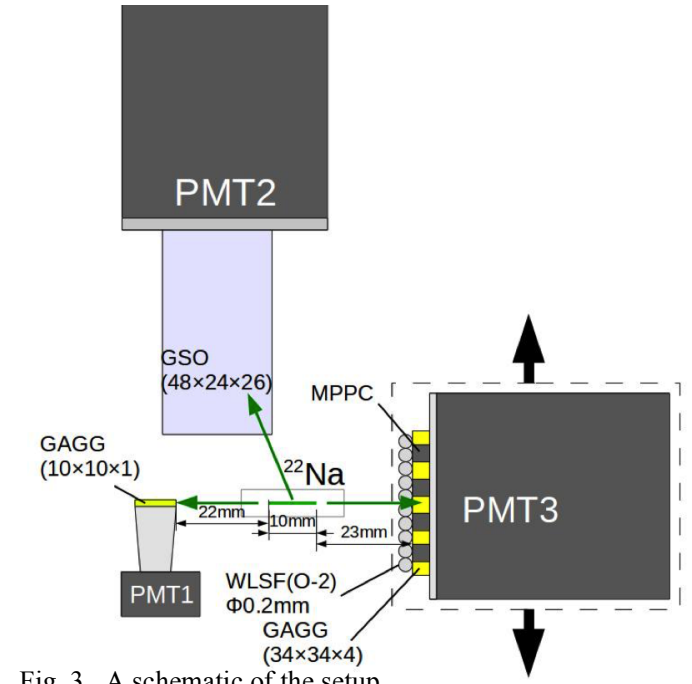

Fig. 3. A schematic of the setup.

of reference consists of a small GAGG scintillator $\left(\mathrm{Gd}_{3} \mathrm{Al}_{2} \mathrm{Ga}_{3} \mathrm{O}_{12}(\mathrm{Ce}), 10 \mathrm{~mm} \times 10 \mathrm{~mm} \times 1 \mathrm{~mm}, \mathrm{C} \& \mathrm{~A}\right.$ Co., Ltd. $)$ and PMT1. The other one consists of GSO $(48 \mathrm{~mm} \times 24 \mathrm{~mm} \times$ 26mm, Asahi Kasei Co., Ltd.) and PMT2. A main detector consists of a big GAGG scintillator $(34 \mathrm{~mm} \times 34 \mathrm{~mm} \times 4 \mathrm{~mm}$, C\&A Co., Ltd.), WLSF[O-2] (Kuraray Co., Ltd.), MPPCs 
(Hamamatsu Photonics Co., Ltd.) and a PMT3 (Hamamatsu Photonics Co., Ltd.). 160 WLSFs are used in the main detector and each 20 fibers are tied and connected to MPPCs. At each side of GAGG, 4 MPPCs are glued.

The small GAGG is at the front of the big GAGG and ${ }^{22} \mathrm{Na}$ shielded source is between them. When reference using PMT1 and the main detector observe energy more than $341 \mathrm{keV}$ as well as reference using PMT2 observe $700 \mathrm{keV}$ simultaneously, PMT1 3 and MPPCs are read signals. Therefor narrow $511-\mathrm{keV}$ gamma beam (its width is approximately $1 \mathrm{~mm}$ ) enter to the main detector. We did measurements shifting position of the main detector $2 \mathrm{~mm}$ apart.

\section{RESULTS AND DISCUSSION}

The relative of energy of two references and distribution of PMT3 is shown in Fig.4.

Events that PMT1 and PMT3 observe 511-keV gamma ray
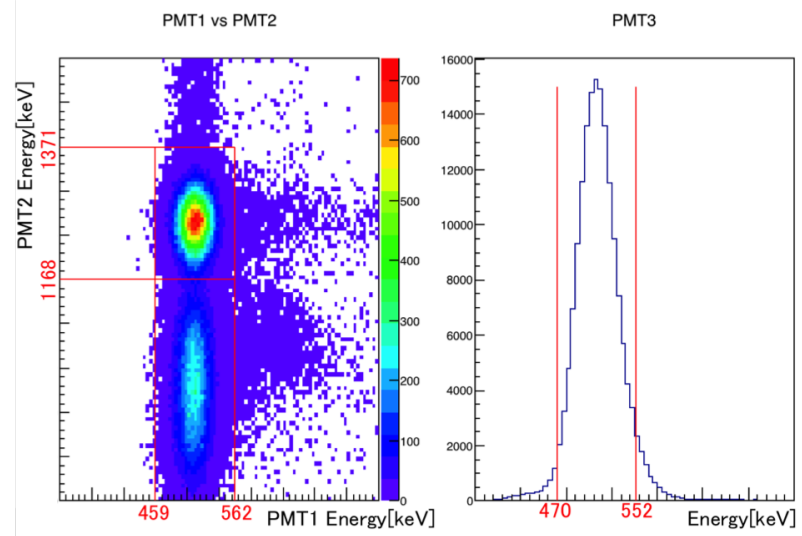

Fig. 4. Distributions of PMTs.

and PMT2 observes 1.27 MeV gamma-ray are selected. Ranges of selection are \pm 2 sigma for each peak.

The total number of photoelectrons of MPPCs connected fibers is $4.34 \pm 0.02$. The incident position is reconstructed by the center of gravity calculation of fiber's position and number of photoelectron of connected MPPC. Fig.5 is distribution of reconstruct position at the incident position of $2 \mathrm{~mm}$.

The relative of reconstruct position and the incident position

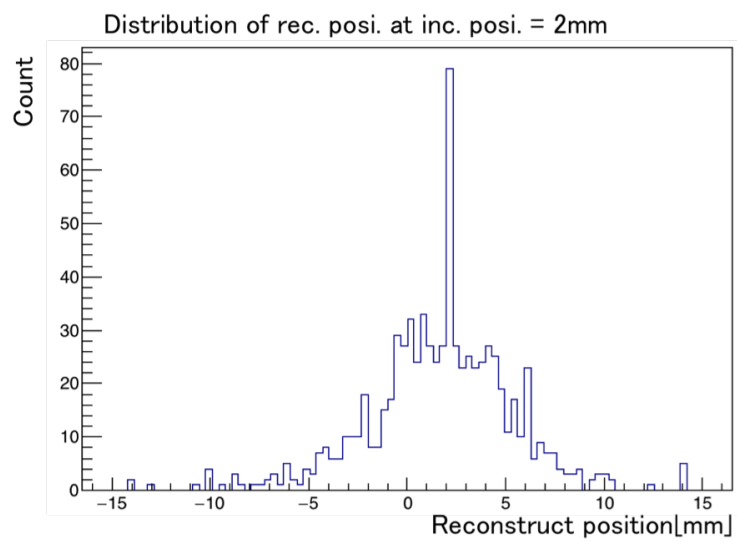

Fig. 5. Distribution of reconstruct position at the incident position of $2 \mathrm{~mm}$.
Rec.position for Inc.position

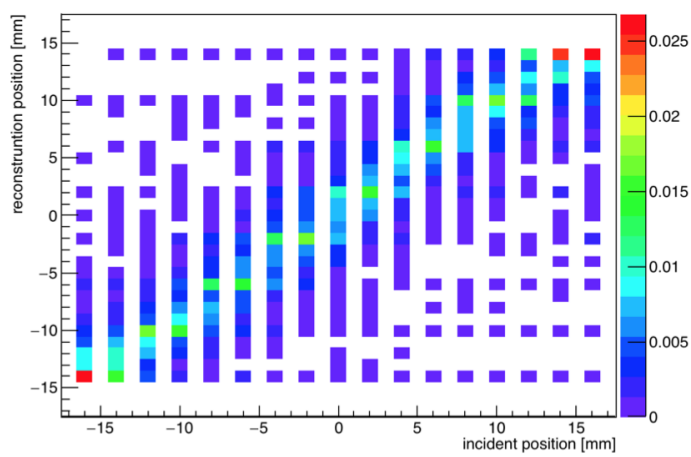

(a)

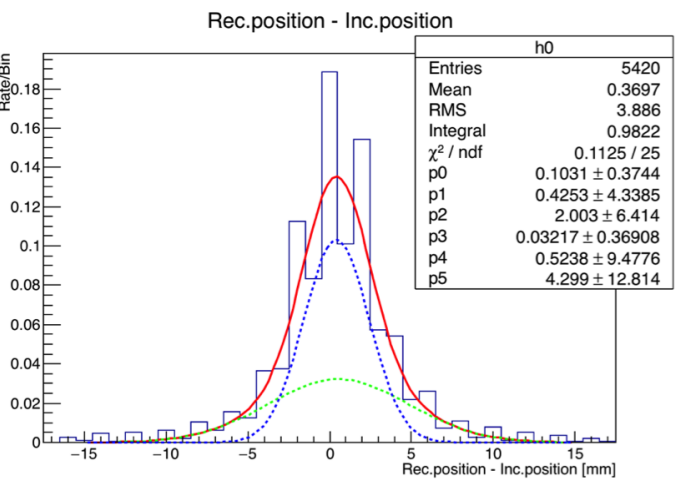

(b)

Fig. 6. Those figures show reconstruction of incident position. (a) A Figure of Reconstruct position vs original position.

(b) A dfference of original position and reconstructed position.

is shown in Fig.6(a). In addition distribution of difference of original incident position and reconstructed position is shown inFig.6(b).

In Fig.6(b), the red line is double Gaussian fitting that is summation of the brue one and green one. The position resolution is calculated $5.4 \mathrm{~mm}$ from red one. In this experiment, each 20 WLSFs are tied. If WLSFs are not tied and read one by one, the position resolution probably achieve less than $1 \mathrm{~mm}$.

Fig.7 is the distribution of total photoelectron of glued MPPCs at side.

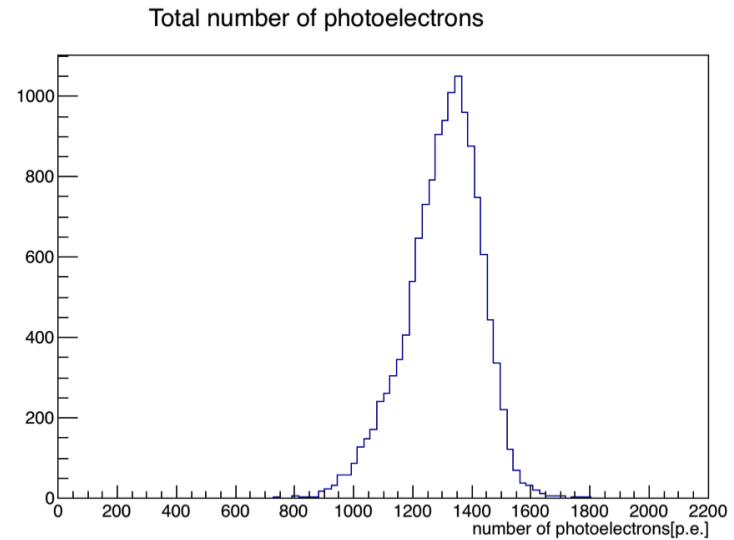

Fig. 7. The distribution of total photoelectron.

The peak of distribution is 1410 p.e.. The energy resolution is also calculated and it is approximately $18.8 \%$ (FWHM). In this experiment, 4 MPPCs are glued on a side of GAGG. It mean 
that effective area of MPPCs cover only $26.5 \%$ of surface. If 10 MPPCs are used and cover $78 \%$ of surface, a number of photoelectrons become two-and-a-half-times. The energy resolution also become less than $12 \%$.

\section{CONCLUSION}

A new PET detector with WLSFs are developed and estimated performance. The position resolution and the energy resolution of demonstration are $5.4 \mathrm{~mm}$ and $18.8 \%$, respectively. In addition, we are conducting more accurate experiment and producing a prototyape detector. Its result also will be shown in this conference.

\section{REFERENCES}

[1] H.Ito, et al., IEEE Medical Imaging Conference 2014, 8-15 Nov. 2014, Seattle, Washington, USA

[2] A.Kobayashi, et al., Advancements in Nuclear Instrumentation Measurement Methods and their Applications, 223, Apr. 20-24 2015, Lisbon Convention Center, Portugal

[3] N.naomi et al., Advancements in Nuclear Instrumentation Measurement Methods and their Applications, 220, Apr. 20-24 2015, Lisbon Convention Center, Portugal 